\title{
Cosmic vector for dark energy: Constraints from supernovae, cosmic microwave background, and baryon acoustic oscillations
}

\author{
Jose Beltrán Jiménez, ${ }^{1}$ Ruth Lazkoz, ${ }^{2}$ and Antonio L. Maroto ${ }^{1}$ \\ ${ }^{1}$ Departamento de Física Teórica, Universidad Complutense de Madrid, 28040 Madrid, Spain. \\ ${ }^{2}$ Fisika Teorikoa, Zientzia eta Teknologiaren Fakultatea, Euskal Herriko Univertsitatea, 644 Posta Kutxatila, 48080 Bilbao, Spain.
} (Received 2 April 2009; published 10 July 2009)

\begin{abstract}
It has been recently shown that the presence of a vector field over cosmological scales could explain the observed accelerated expansion of the Universe without introducing either new scales or unnatural initial conditions in the early Universe, thus avoiding the coincidence problem. Here, we present a detailed analysis of the constraints imposed by supernova type Ia (SNIa), cosmic microwave background (CMB), and baryon acoustic oscillation (BAO) data on the vector dark energy model with general spatial curvature. We find that contrary to standard cosmology, CMB data exclude a flat universe for this model and, in fact, predict a closed geometry for the spatial sections. We see that CMB and SNIa Gold data are perfectly compatible at the 1-sigma level, however the SNIa Union data set exhibits a 3-sigma tension with CMB. The same level of tension is also found between SNIa and BAO measurements.
\end{abstract}

DOI: 10.1103/PhysRevD.80.023004

PACS numbers: 95.36.+x, 98.80.-k, 98.80.Es

\section{INTRODUCTION}

More than ten years have passed since the first indications of the accelerated expansion of the Universe [1] and still today it remains as one of the most intriguing problems in cosmology. Moreover, the accelerated expansion has been confirmed during the last decade by many different probes, mainly through measurements of type Ia supernovae (SNIa), cosmic microwave background (CMB) temperature power spectrum, and the baryon acoustic oscillations (BAO) [2-4]. Since Einstein's gravity predicts that a universe containing (baryonic and dark) matter and radiation should be decelerating rather than accelerating, these observations could be signalling either the breakdown of general relativity at cosmological scales or the existence of some sort of nonordinary energy with negative pressure known as dark energy.

The simplest model describing dark energy is the existence of a cosmological constant. Although it fits observations with very good precision, it suffers from theoretical problems since the inferred scale for the cosmological constant turns out to be around $10^{-3} \mathrm{eV}$, which is a very tiny value compared to the gravitational scale set by Newton's constant $G \simeq M_{P}^{-2}$ with $M_{P} \sim 10^{19} \mathrm{GeV}$. This poses a problem of naturalness because of the existence of two scales in the theory which differ in many orders of magnitude. Moreover, a related problem arises because the amount of energy density stored in the form of dark energy is comparable to that stored in the form of matter at the present epoch, in spite of having evolved very differently in the past. Thus, in order to get around these problems, several models have been proposed which are mostly based on either cosmological scalar fields $[5,6]$ or infrared modifications of the gravitational action [7,8] (see [9] for a review on dark energy models). However, none of these models succeeded in solving the previously addressed problems of the cosmological constant because they either introduce new dimensional scales in the action or unnatural initial conditions to get the right acceleration at the right time.

In a previous paper [10] we showed that a model based on the dynamics of a vector field on cosmological scales can give rise to a period of accelerated expansion without introducing either new dimensional scales or unnatural initial conditions, thus avoiding fine-tuning or coincidence problems. Although the existence of periods of accelerated expansion for vector field models was already known $[11,12]$, the model proposed in [10] consists just of the simplest kinetic terms for the vector field containing two fields and two derivatives and without any potential for the field. In fact, in this paper we shall show that such a model is nothing but a gauge field with a gauge-fixing term coupled to the Ricci tensor. Moreover, very recently [13] it has been also shown that the electromagnetic field itself could be a natural candidate for dark energy.

In order to constrain dark energy models from observations, we typically use distance indicators so that we can confront distance measurements to the corresponding model predictions [14]. To do that we can resort to two different types of objects, namely standard candles and standard rulers. Standard candles are objects of known intrinsic luminosity, so that the corresponding comoving distance can be determined. That way, it is possible to reconstruct the Hubble expansion rate by searching this sort of object at different redshifts. The most important class of such indicators is type Ia supernovae. On the other hand, standard rulers are objects whose comoving size is known, so that we can measure the angular distance and, therefore, compare to that predicted by the dark energy model. A well-known example is the sound horizon size at the last scattering surface. This standard ruler can be measured directly from the CMB temperature power spec- 
trum and, also, from baryon acoustic oscillations (BAO) through the matter power spectrum at low redshift.

The paper is organized as follows: in Sec. I we introduce the vector model for dark energy and derive all the necessary equations for the rest of the paper. In Sec. II we show how the different distance indicators will be used in order to obtain constraints for the model. Finally, Sec. III is devoted to the results obtained from the analysis.

\section{VECTOR DARK ENERGY}

The action proposed in [10] to describe dark energy is the following:

$$
\begin{aligned}
S= & \int d^{4} x \sqrt{-g}\left(-\frac{R}{16 \pi G}-\frac{1}{2} \nabla_{\mu} A_{\nu} \nabla^{\mu} A^{\nu}\right. \\
& \left.+\frac{1}{2} R_{\mu \nu} A^{\mu} A^{\nu}\right) .
\end{aligned}
$$

However, this action can be written in a more suggestive form by integrating by parts and taking into account that $R_{\mu \nu} A^{\mu} A^{\nu}=\left(\nabla_{\mu} A^{\mu}\right)^{2}-\nabla_{\mu} A_{\nu} \nabla^{\nu} A^{\mu}$. Then, the action for the vector field can be expressed as

$$
\begin{aligned}
S= & \int d^{4} x \sqrt{-g}\left[-\frac{R}{16 \pi G}-\frac{1}{4} F_{\mu \nu} F^{\mu \nu}-\frac{1}{2}\left(\nabla_{\mu} A^{\mu}\right)^{2}\right. \\
& \left.+R_{\mu \nu} A^{\mu} A^{\nu}\right] .
\end{aligned}
$$

In this form, it becomes apparent that the theory is that of a gauge vector field with a gauge-fixing term (in the Feynman gauge) and coupled to the Ricci tensor. This coupling provides an effective mass term driven by gravity.

Now, we take variations with respect to the metric and the vector field to obtain the Einstein and vector field equations respectively:

$$
\begin{gathered}
R_{\mu \nu}-\frac{1}{2} R g_{\mu \nu}=8 \pi G\left(T_{\mu \nu}+T_{\mu \nu}^{A}\right), \\
\nabla_{\nu} \nabla^{\nu} A_{\mu}+R_{\mu \nu} A^{\nu}=0,
\end{gathered}
$$

where $T_{\mu \nu}$ is the conserved energy-momentum tensor for matter and radiation (and all other possible components) and $T_{\mu \nu}^{A}$ is the energy-momentum tensor for the vector field. We consider the case in which the vector field is homogeneous and only has time component, i.e., $A_{\mu}=$ $\left(A_{0}(t), 0,0,0\right)$. However, unlike in the previous paper [10], here we will consider the effects of the curvature so the metric is given by

$$
d s^{2}=d t^{2}-a(t)^{2}\left[\frac{d r^{2}}{1-k r^{2}}+r^{2}\left(d \theta^{2}+\sin ^{2} \theta d \phi^{2}\right)\right] .
$$

In this metric, Eq. (4) for the homogeneous time component of the vector field reads

$$
\ddot{A}_{0}+3 H \dot{A}_{0}-3\left(2 H^{2}+\dot{H}\right) A_{0}=0,
$$

where $H=\dot{a} / a$ is the Hubble parameter. For our purposes on this work it will be useful to express the latter equation in terms of the redshift $z=\frac{1}{a}-1$ as follows:

$$
\frac{d^{2} A_{0}}{d z^{2}}+\frac{1}{(1+z) H(z)} \frac{d}{d z}\left[\frac{H(z)}{(1+z)^{2}}\right] \frac{d}{d z}\left[(1+z)^{3} A_{0}\right]=0 .
$$

This equation can be easily solved when the Universe is dominated by radiation or matter, assuming that the contribution of the vector field is negligible. In those epochs the Hubble parameter is given by $H=p / t=p(1+z)^{1 / p}$ with $p=1 / 2$ for radiation and $p=2 / 3$ for matter and, according to (7), the vector field evolves as

$$
A_{0}(z)=A_{0}^{+}(1+z)^{\alpha_{+}}+A_{0}^{-}(1+z)^{\alpha_{-}}
$$

with $A_{0}^{ \pm}$constants of integration and $\alpha_{ \pm}=(1 \pm 1) / 2$ in the radiation era, and $\alpha_{ \pm}=(3 \pm \sqrt{33}) / 4$ in the matter era. Notice that, since the vector field is constant during the radiation era, we can set the initial conditions at any time during that epoch without modifying the evolution of the Universe.

On one hand, the (00) component of Einstein's equations can be written as

$$
\frac{H^{2}}{H_{0}^{2}}=\Omega_{m}(1+z)^{3}+\Omega_{r}(1+z)^{4}+\Omega_{k}(1+z)^{2}+\rho_{A}(z),
$$

where $H_{0}$ is the present value of the Hubble parameter, which is usually expressed as $H_{0}=100 \mathrm{hm} \mathrm{s}^{-1} \mathrm{Mpc}^{-1}$, $\Omega_{m}$ and $\Omega_{r}$ are the density parameters corresponding to matter and radiation, respectively, $\Omega_{k}=-\frac{k}{H_{0}^{2}}$, and $\rho_{A}$ is the energy density of the vector field, whose expression is

$$
\rho_{A}=\frac{H^{2}}{H_{0}^{2}}\left[\frac{1}{2} A_{0}^{2}-(1+z) A_{0} \frac{d A_{0}}{d z}-\frac{1}{6}(1+z)^{2}\left(\frac{d A_{0}}{d z}\right)^{2}\right] .
$$

Moreover, we measure the vector field in units of the reduced Planck mass $\tilde{M}_{P}=1 / \sqrt{8 \pi G}$. We can obtain the evolution of this energy density in the radiation and matter dominated eras by using the growing solution in (8) for the vector field to give

$$
\rho_{A}=\rho_{A 0}(1+z)^{\kappa},
$$

where $\kappa=4$ in the radiation era and $\kappa=(9-\sqrt{33}) / 2 \simeq$ 1.63 in the matter era. Notice that the energy density scales as radiation in the early Universe so that $\rho_{A} / \rho_{R}$ is constant in that epoch (see in Fig. 1). Therefore, we obtain once again that the time at which we set the initial conditions in the early Universe for the vector field lacks importance, since its fraction of energy density is constant during that epoch.

Finally, when the vector field becomes dominant the Universe suffers a type III singularity [15] in which its 

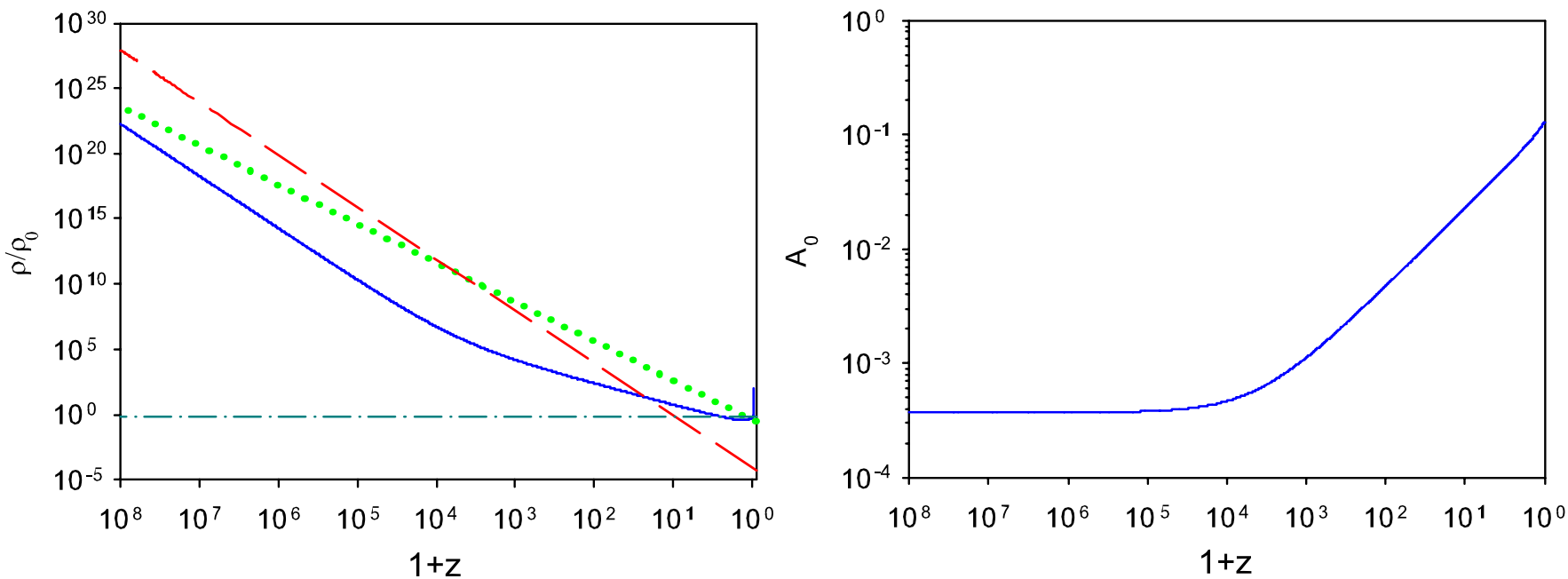

FIG. 1 (color online). Left panel: evolution of energy densities. Dashed (red) for radiation, dotted (green) for matter, and solid (blue) for vector dark energy. We also show for comparison the cosmological constant energy density in dash-dotted line. We see the rapid growth of dark energy contribution at late times approaching the final singularity. Right panel: cosmological evolution of the vector field. In these two plots we see the unimportance of the time at which we set the initial conditions due to the fact that both the vector field and the fraction of dark energy density are constant in the early Universe.

evolution ends at a finite time $t_{\text {end }}$ but with a finite size $a_{\text {end }}$. Moreover, as we approach the final singularity we have $\rho_{\mathrm{DE}} \rightarrow \infty$ and $p_{\mathrm{DE}} \rightarrow-\infty$, with $w_{\mathrm{DE}} \rightarrow-\infty$, whereas the vector field takes a finite value.

On the other hand, the (ii) component of Einstein's equations is

$$
\begin{aligned}
\frac{H^{2}}{H_{0}^{2}}\left[3-\frac{1+z}{H} \frac{d H}{d z}\right]= & \Omega_{r}(1+z)^{4}+\Omega_{k}(1+z)^{2} \\
& -p_{A}(z)
\end{aligned}
$$

where we have used that $p_{r}=\frac{1}{3} \rho_{r}$ and $p_{m}=0$ and the pressure of the vector field is given by

$$
\begin{aligned}
p_{A}(z)= & -\frac{H^{2}}{H_{0}^{2}}\left\{3\left[\frac{5}{2}-\frac{4}{3}(1+z) \frac{1}{H} \frac{d H}{d z}\right] A_{0}^{2}\right. \\
& \left.+(1+z) A_{0} \frac{d A_{0}}{d z}+\frac{3}{2}(1+z)^{2}\left(\frac{d A_{0}}{d z}\right)^{2}\right\} .
\end{aligned}
$$

In order to perform the analysis in next sections it will be convenient to write Eqs. (9) and (12) in terms of $\left\{\Omega_{r} h^{2}, \Omega_{m} h^{2}, \Omega_{k} h^{2}\right\}$ as follows:

$$
\begin{aligned}
\hat{H}^{2}= & \Omega_{m} h^{2}(1+z)^{3}+\Omega_{r} h^{2}(1+z)^{4}+\Omega_{k} h^{2}(1+z)^{2} \\
& +\rho_{A}(z), \\
\hat{H}\left[3-\frac{1+z}{\hat{H}} \frac{d \hat{H}}{d z}\right]= & \Omega_{r} h^{2}(1+z)^{4}+\Omega_{k} h^{2}(1+z)^{2} \\
& -p_{A}(z),
\end{aligned}
$$

where $\hat{H} \equiv H /\left(100 \mathrm{~km} \mathrm{~s}^{-1} \mathrm{Mpc}^{-1}\right)$, i.e., $\hat{H}(z=0)=h$. Note that neither $\rho_{A}$ nor $p_{A}$ depend on the normalization of the Hubble parameter. Moreover, $\Omega_{r} h^{2}$ contains the contribution of photons as long as relativistic neutrinos

$$
\Omega_{r} h^{2}=\Omega_{\gamma} h^{2}\left(1+0.2271 N_{\mathrm{eff}}\right)
$$

with $N_{\text {eff }}=3.04$ the effective number of neutrino species and $\Omega_{\gamma} h^{2}=2.469 \times 10^{-5}$ for $T_{\mathrm{CMB}}=2.725$.

The model is completely determined once we fix the set of parameters $\left\{\Omega_{m}, \Omega_{k}, A_{\text {rad }}\right\}$, where $A_{\text {rad }}$ is the constant value of $A_{0}$ during radiation era, so that the model has three free parameters. To confront the model to $\mathrm{SN}$ and BAO data set we only need to integrate the system of equations up to redshift $\sim 2$ whereas $\mathrm{CMB}$ data set requires one to obtain the solution up to the last scattering surface so that the method to solve the equations will be different for each case. The present value of the Hubble expansion rate is no longer a free parameter in this model because it can be obtained in terms of the previous parameters after integrating the equations. In fact, we could take $\left\{\Omega_{m}, \Omega_{k}, h\right\}$ as independent parameters and, therefore, $A_{\text {rad }}$ would already be determined, although this approach is more difficult to implement numerically. Notice that this model contains exactly the same number of parameters as $\Lambda \mathrm{CDM}$.

\section{LIKELIHOOD CALCULATIONS}

In this section we shall explain the procedure followed to confront the vector dark energy model to the different distance indicators.

\section{A. SN}

The apparent magnitude of a supernova placed at a given redshift $z$ is related to the expansion history of the Universe through the distance modulus 


$$
\mu \equiv m-M=5 \log D_{L}-5 \log h+\mu_{0},
$$

where $m$ and $M$ are the apparent and absolute magnitudes, respectively, $\mu_{0}=42.38$ and $D_{L}=H_{0} d_{L}$ with $d_{L}$ the luminosity distance $d_{L}=(1+z) r(z)$, being $r(z)$ the comoving distance, given for the metric (5) by

$$
r(z)=\frac{1}{H_{0} \sqrt{\left|\Omega_{k}\right|}} S_{k}\left[\sqrt{\left|\Omega_{k}\right|} \int_{0}^{z} \frac{H_{0}}{H\left(z^{\prime}\right)} d z^{\prime}\right]
$$

with $S_{k}[x]=\sin x, x, \sinh x$ for $\Omega_{k}<0, \Omega_{k}=0, \Omega_{k}>0$, respectively.

Then, to confront the model to each supernovae data set we construct the corresponding $\chi^{2}$ estimator

$$
\chi_{\mathrm{SN}}^{2}=\sum_{i=1}^{N} \frac{\left(\mu\left(z_{i} ; \Omega_{m}, \Omega_{k}, h\right)-\mu_{i}\right)^{2}}{\sigma_{i}^{2}},
$$

which must be marginalized over $h$ in order to obtain the constraints on the parameters $\Omega_{m}$ and $\Omega_{k}$.

In order to calculate $\chi_{\mathrm{SN}}^{2}$, we use the fact that the SNIa data set corresponds to redshifts below 2 so that we can neglect the contribution from radiation in Einstein's equations. With this in mind, we solve numerically the system of Eqs. (7) and (12) for $H / H_{0}$ and $A_{0}$. As this system is of second order with respect to $A_{0}$ and first order with respect to $H / H_{0}$ we need to set the initial values of $A_{0}, d A_{0} / d z$, and $H / H_{0}$. However, these three initial values are related by means of the Friedmann equation (9) so that we can obtain the initial value for $H / H_{0}$ in terms of the initial values of $A_{0}$ and its derivative. On the other hand, as we know the analytic solution of the vector field in the matter dominated era as that given in (8), we can relate the initial value of the derivative of the vector field to the initial value of the vector field (neglecting the decaying mode). Therefore, we only need to give the initial value for $A_{0}$ in order to set the initial conditions and we are left with $A_{0}^{\text {ini }}$, $\Omega_{m}$, and $\Omega_{k}$ as free parameters in terms of which we obtain the corresponding $\chi_{\mathrm{SN}}^{2}$ estimator. Therefore, we shall use

$$
\chi_{\mathrm{SN}}^{2}=\sum_{i=1}^{N} \frac{\left(\mu\left(z_{i} ; \Omega_{m}, \Omega_{k}, A_{\mathrm{ini}}\right)-\mu_{i}\right)^{2}}{\sigma_{i}^{2}}
$$

instead of (19) and marginalize over $A_{\text {ini }}$.

In this work we have used two sets of supernovae: the Gold set [2] and the more recent Union set [3].

\section{B. BAO}

$\mathrm{BAO}$ measurements provide the following distance ratios [16]:

$$
\mathbf{V}_{\mathrm{BAO}} \equiv\left(\begin{array}{c}
\frac{r_{s}\left(z_{d}\right)}{D_{V^{\prime}(0.2)}} \\
\frac{r_{s}\left(z_{d}\right)}{D_{V}(0.35)}
\end{array}\right)=\left(\begin{array}{c}
0.1980 \pm 0.0058 \\
0.1094 \pm 0.0033
\end{array}\right)
$$

where $r_{s}(z)$ is the sound horizon size given by

$$
r_{s}(z)=\frac{1}{\sqrt{3}} \int_{0}^{1 /(1+z)} \frac{d a}{a^{2} H(a) \sqrt{\left(1+\frac{3 \Omega_{b} h^{2}}{4 \Omega_{\gamma} h^{2}} a\right)}}
$$

and

$$
D_{V}(z)=\left[r^{2}(z) \frac{z}{H}\right]^{1 / 3}
$$

is the dilation scale. Finally, $z_{d}$ is the drag epoch at which baryons were released from photons and which can be calculated by using the fitting formula [17]

$$
z_{d}=\frac{1291\left(\Omega_{m} h^{2}\right)^{0.251}}{1+0.659\left(\Omega_{m} h^{2}\right)^{0.828}}\left[1+b_{1}\left(\Omega_{b} h^{2}\right)^{b_{2}}\right]
$$

with

$$
\begin{gathered}
b_{1}=0.313\left(\Omega_{m} h^{2}\right)^{-0.419}\left[1+0.607\left(\Omega_{m} h^{2}\right)^{0.674}\right], \\
b_{2}=0.238\left(\Omega_{m} h^{2}\right)^{0.223} .
\end{gathered}
$$

Then, we define the BAO array

$$
\mathbf{X}_{\mathrm{BAO}} \equiv\left(\begin{array}{c}
\frac{r_{s}\left(z_{d}\right)}{D_{V}(0.2)}-1.980 \\
\frac{r_{s}\left(z_{d}\right)}{D_{V}(0.35)}-0.1094
\end{array}\right),
$$

so that

$$
\chi_{\mathrm{BAO}}^{2}=\mathbf{X}_{\mathrm{BAO}}^{T} \mathbf{C}_{\mathrm{BAO}}^{-1} \mathbf{X}_{\mathrm{BAO}} .
$$

In this expression, the inverse covariance matrix is

$$
\mathbf{C}_{\mathrm{BAO}}^{-1}=\left(\begin{array}{cc}
35059 & -24031 \\
-24031 & 108300
\end{array}\right) .
$$

The procedure we follow in this case is analogous to that used for the SNIa analysis, although, as $\chi_{\mathrm{BAO}}^{2}$ depends on the amount of baryons $\Omega_{b}$, we also need to marginalize over this parameter.

\section{C. $\mathrm{CMB}$}

Following [18], we use the distance priors method to confront dark energy models to CMB data [19]. This method uses two distance ratios measured by means of the CMB temperature power spectrum:

(i) The "acoustic scale," which measures the ratio of the angular diameter distance to the decoupling epoch and the comoving sound horizon size at decoupling epoch. This first distance ratio can be expressed as

$$
l_{A} \equiv \frac{\pi r\left(z_{*}\right)}{r_{s}\left(z_{*}\right)} .
$$

Moreover, we use the fitting formula of $z_{*}$ proposed in [20]

$$
\begin{aligned}
z_{*}= & 1048\left[1+0.00124\left(\Omega_{b} h^{2}\right)^{-0.738}\right] \\
& \times\left[1+g_{1}\left(\Omega_{m} h^{2}\right)^{g_{2}}\right]
\end{aligned}
$$


with

$$
\begin{aligned}
& g_{1}=\frac{0.0783\left(\Omega_{b} h^{2}\right)^{-0.238}}{1+39.5\left(\Omega_{b} h^{2}\right)^{0.763}}, \\
& g_{2}=\frac{0.560}{1+21.1\left(\Omega_{b} h^{2}\right)^{1.81}},
\end{aligned}
$$

(ii) The second distance ratio measures the ratio of the angular diameter distance and the Hubble ratio at the decoupling time. It is usually called the "shift parameter" and can be expressed as

$$
R=\sqrt{\Omega_{m} H_{0}^{2}} r\left(z_{*}\right) .
$$

The values reported in [18] for these distance priors are

$$
\mathbf{V}_{\mathrm{CMB}} \equiv\left(\begin{array}{c}
l_{A}\left(z_{*}\right) \\
R\left(z_{*}\right) \\
z_{*}
\end{array}\right)=\left(\begin{array}{c}
302.10 \pm 0.86 \\
1.710 \pm 0.019 \\
1090.04 \pm 0.93
\end{array}\right)
$$

with the following inverse of the covariance matrix:

$$
\mathbf{C}_{\mathrm{CMB}}^{-1}=\left(\begin{array}{ccc}
1.800 & 27.968 & -1.103 \\
27.968 & 5667.577 & -92.263 \\
-1.103 & -92.263 & 2.923
\end{array}\right) .
$$

Then, we define the CMB array as

$$
\mathbf{X}_{\mathrm{CMB}}=\left(\begin{array}{c}
l_{A}-302.10 \\
R-1.710 \\
z_{*}-1090.04
\end{array}\right)
$$

so that

$$
\chi_{\mathrm{CMB}}^{2}=\mathbf{X}_{\mathrm{CMB}}^{T} \mathbf{C}_{\mathrm{CMB}}^{-1} \mathbf{X}_{\mathrm{CMB}} .
$$

The procedure we follow in this case is somewhat different from that used in the previous sections. The main difference comes from the fact that $\mathrm{CMB}$ distance priors are evaluated at a time when radiation is important so that we cannot neglect its contribution in Einstein's equations anymore. To simplify numerical calculations we use Eqs. (7), (14), and (15). Notice that, unlike the SN and $\mathrm{BAO}$ approach, from these equations we obtain the Hubble expansion rate normalized to $100 \mathrm{~km} \mathrm{~s}^{-1} \mathrm{Mpc}^{-1}$ so that $\hat{H}(z=0)=h$. Thus, for given $\left\{\Omega_{m} h^{2}, \Omega_{k} h^{2}\right\}$ we use (14) to relate the initial condition for the Hubble expansion rate to the initial condition of the vector field (that we shall name $A_{\text {rad }}$ ) and, then, solve numerically (7) and (15). Since the initial conditions are set in the radiation-dominated era when, according to (8), the vector field is constant, the initial condition for the derivative of the vector field is set to zero. Moreover, the constancy of the vector field during that epoch eliminates the dependency on the time at which we place the initial conditions, i.e., $A_{\text {rad }}$ does not depend on $z_{\text {ini }}$. That way, we obtain the expansion rate $\hat{H}(z)$ that will allow us to compute the distance indicators described above in terms of $\left\{\Omega_{m} h^{2}, \Omega_{k} h^{2}, A_{\text {rad }}\right\}$ (notice that such indicators do not depend on the normalization of the Hubble expansion rate). Hence, we can compute the corresponding $\chi_{\mathrm{CMB}}^{2}$ which will depend on $\left\{\Omega_{m} h^{2}, \Omega_{k} h^{2}\right.$, $\left.\Omega_{b} h^{2}, A_{\text {rad }}\right\}$ and, following the prescription given in [18], we marginalize over $\Omega_{b} h^{2}$ and $A_{\text {rad }}$ (which is equivalent to marginalizing over $h$ ) and use the resulting marginalized likelihood to obtain the corresponding contours.

Since CMB distance priors were derived in [18] assuming that dark energy was not important at decoupling time $\left(z_{*} \simeq 1090\right)$ and given that the vector field model does not produce a significant amount of dark energy at high redshifts, these priors are, in principle, applicable in this case.

\section{RESULTS}

In this section we present the results obtained after confronting the model given by the action (2) with the tests explained above. We have also performed the analysis for a $\Lambda \mathrm{CDM}$ model for comparison.

Using the Gold data set we obtain a best fit for $\Omega_{m}=$ 0.385 and $\Omega_{A}=0.611$ with $\chi_{\min }^{2}=172.92$, which is the same value found in [10] where we imposed flat spatial sections. This is understandable because, from the above values of $\Omega_{m}$ and $\Omega_{A}$, we obtain $\Omega_{k}=0.0043$ so that the best fit is very close to the flat case. However the $1 \sigma$ contour allows both open and closed universes and, unlike [10], a wide range of values for $\Omega_{m}$ and $\Omega_{A}$ is within the $1 \sigma$ region, as we can see in Fig. 2 . For a $\Lambda$ CDM model with nonvanishing curvature we obtain the best fit for $\Omega_{m}=$ 0.46 and $\Omega_{\Lambda}=0.98$ with $\chi_{\min }^{2}=175.04$ so we still obtain a better fit to the Gold data set than $\Lambda$ CDM. On the other hand, the best fit obtained for the vector dark energy model from the Union data set corresponds to $\Omega_{m}=0.260$ and $\Omega_{A}=0.503$ with $\chi_{\min }^{2}=311.96$. From Fig. 2 we see that this data set favors an open universe for this model, being the flat case at more than $2 \sigma$. For $\Lambda \mathrm{CDM}$ the best fit happens for $\Omega_{m}=0.41$ and $\Omega_{\Lambda}=0.93$, being $\chi_{\min }^{2}=$ 310.23 , which is lower than that obtained for the vector field. This effect is probably due to the Supernova Legacy Survey points contained in the Union data set which, as it is shown in [10], favor $\Lambda \mathrm{CDM}$ over the vector field model at more than $2 \sigma$ in the flat case. However, when the flatness assumption is dropped, $\Lambda \mathrm{CDM}$ fits the Union data set better than the vector field model only at less than $1 \sigma$.

Concerning BAO data set, it favors an open universe with a small amount of matter for the vector field model, as we see in Fig. 2. Moreover, the compatibility of these data with SNIa data is only at the $3 \sigma$ level. However, it is worth mentioning that these distance indicators are obtained after analyzing the actual observational data with $\Lambda \mathrm{CDM}$ as fiducial model so that its applicability to test dark energy models is justified as long as such models do not differ 

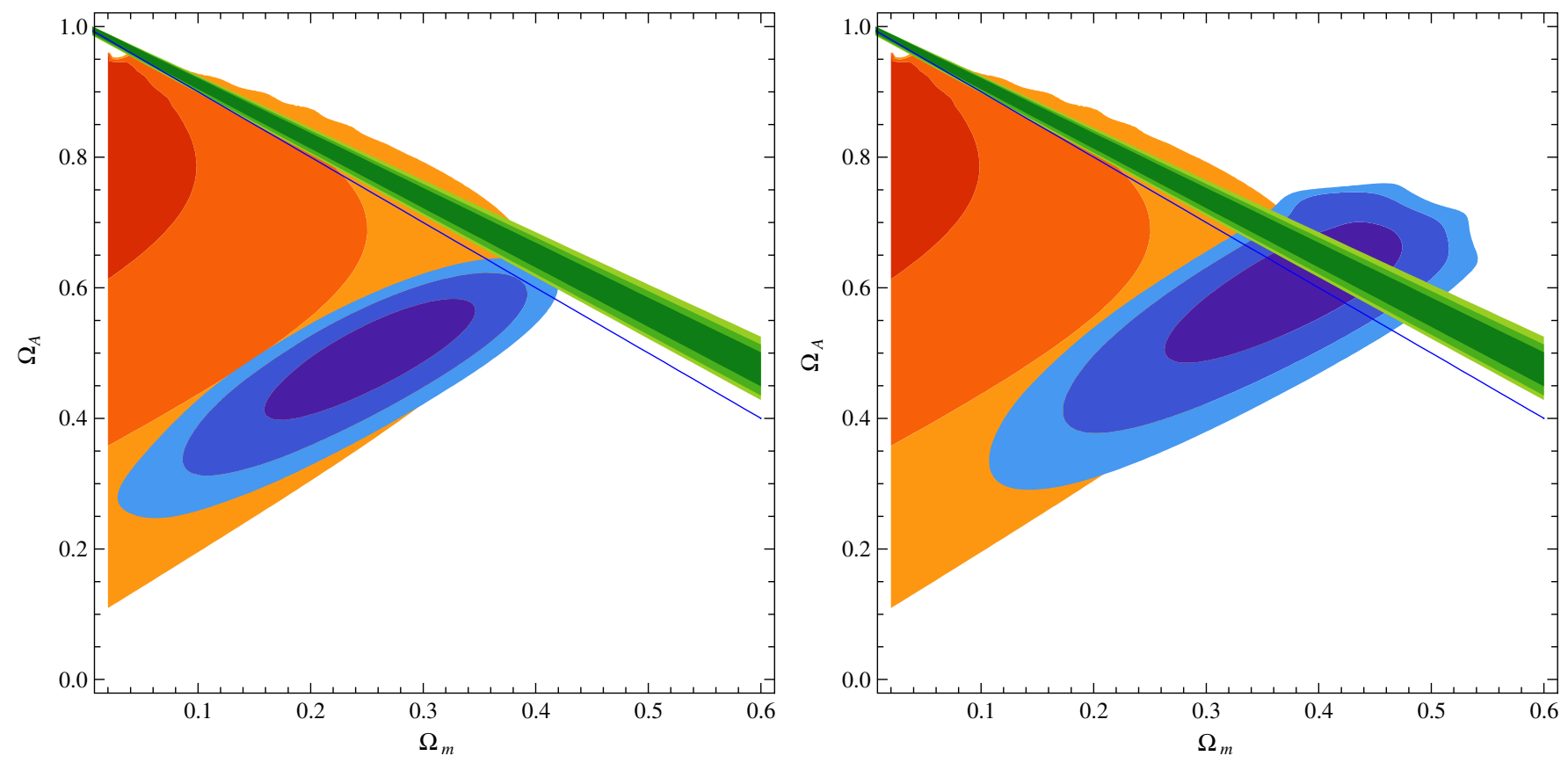

FIG. 2 (color online). In these plots we show the $68 \%, 95 \%$, and 99\% C.L. regions for BAO (large orange regions on the left part of plots), CMB (long green regions in the upper part), and SNIa (closed blue regions in the center). We show the contours obtained for both the Union data set (left) and the Gold data set (right). The blue line corresponds to a flat universe.

much from a cosmological constant. Nevertheless, this is not the case for the vector dark energy model whose equation of state varies very rapidly and, indeed, has a future singularity so that the obtained $3 \sigma$ tension could be due to the dependency of BAO data on the fiducial model. In any case, this is the less confident data set to constrain the vector model and, in general, any dark energy model, since it may give shifted parameters due to a biased determination of the sound horizon scale due to the presence of

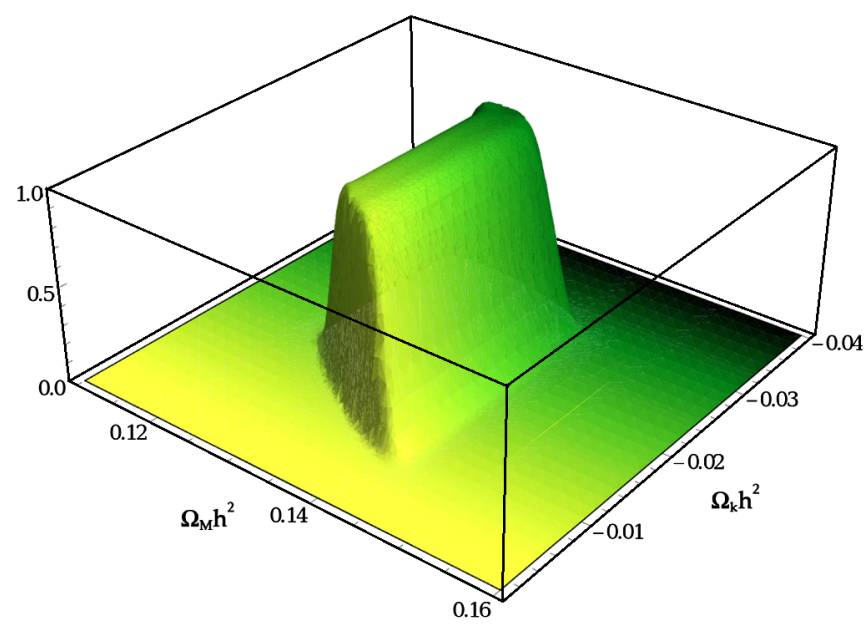

FIG. 3 (color online). In this plot we show the likelihood obtained from the CMB data set for the vector field model. We can see that a flat universe is clearly ruled out and a closed geometry for the spatial sections is strongly favored. additional relativistic degrees of freedom, early dark energy, or a nonstandard recombination scheme [21].

Finally, CMB data are totally incompatible with flat spatial sections and, in fact, they predict a closed universe with a wide range of $\Omega_{m}$ allowed. These results show that, contrary to common belief, CMB data do not necessarily favor a flat universe. In Fig. 3, the corresponding likelihood for the CMB data set is plotted and we can see how the flat case is ruled out for the vector model.

In Fig. 2 we see that CMB contours are compatible with BAO at $2 \sigma$ level for small values of $\Omega_{m}$ and $\Omega_{k}$ close to zero. Concerning SNIa contours, CMB is in conflict with the Union data set contours at more than $3 \sigma$ whereas it is compatible at $1 \sigma$ level with the Gold data set.

\section{CONCLUSIONS}

In this work we have performed a detailed analysis of the constraints imposed by SNIa, CMB, and BAO data on the vector dark energy model proposed in [10]. We have considered cosmologies with arbitrary spatial curvature and obtained confidence regions in the $\left(\Omega_{m}, \Omega_{A}\right)$ plane. We have found that for the SNIa Gold data set, the vector model fit is better than that of $\Lambda \mathrm{CDM}$, but for the Union data set the situation is reversed. We find that contrary to standard cosmology, CMB data exclude a flat universe for this model and, in fact, predict a closed spatial geometry. On the other hand, CMB and SNIa Gold data are perfectly compatible at the 1-sigma level, however SNIa Union data set exhibits a 3-sigma tension with CMB. The same level of tension is also found between SNIa and BAO measure- 
ments, although this may be due to the dependency of BAO measurements on the fiducial model.

\section{ACKNOWLEDGMENTS}

We would like to thank Eiichiro Komatsu for useful comments. J. B. is very grateful to the Department of
Fisika Teorikoa of the EHU for their hospitality. This work has been supported by DGICYT (Spain) Project Nos. FPA 2004-02602 and FPA 2005-02327, UCMSantander PR34/07-15875, CAM/UCM 910309, and MEC grant BES-2006-12059.
[1] A. G. Riess et al., Astron. J. 116, 1009 (1998); S. Perlmutter et al., Astrophys. J. 517, 565 (1999).

[2] A. G. Riess et al., Astrophys. J. 607, 665 (2004).

[3] M. Kowalski et al., Astrophys. J. 686, 749 (2008).

[4] D. N. Spergel et al., Astrophys. J. Suppl. Ser. 148, 175 (2003); 170, 377 (2007); M. Tegmark et al., Phys. Rev. D 69, 103501 (2004).

[5] C. Wetterich, Nucl. Phys. B302, 668 (1988); R. R. Caldwell, R. Dave, and P. J. Steinhardt, Phys. Rev. Lett. 80, 1582 (1998).

[6] C. Armendariz-Picon, T. Damour, and V. Mukhanov, Phys. Lett. B 458, 209 (1999).

[7] S. M. Carroll, V. Duvvuri, M. Trodden, and M. S. Turner, Phys. Rev. D 70, 043528 (2004).

[8] G. Dvali, G. Gabadadze, and M. Porrati, Phys. Lett. B 485, 208 (2000).

[9] E. J. Copeland, M. Sami, and S. Tsujikawa, Int. J. Mod. Phys. D 15, 1753 (2006).

[10] J. Beltrán Jiménez and A.L. Maroto, Phys. Rev. D 78, 063005 (2008); arXiv:0807.2528.

[11] V. V. Kiselev, Classical Quantum Gravity 21, 3323 (2004).

[12] C. Armendariz-Picon, J. Cosmol. Astropart. Phys. 07 (2004) 007; C. G. Boehmer and T. Harko, Eur. Phys. J. C 50, 423 (2007); M. Novello et al., Phys. Rev. D 69,
127301 (2004); T. Koivisto and D. F. Mota, Astrophys. J. 6791 (2008).

[13] J. Beltrán Jiménez and A. L. Maroto, J. Cosmol. Astropart. Phys. 03 (2009) 016; arXiv:0903.4672; J. Cosmol. Astropart. Phys. 02 (2009) 025; AIP Conf. Proc. 1122, 107 (2009).

[14] E. L. Wright, Astrophys. J. 664, 633 (2007); R. Lazkoz, S. Nesseris, and L. Perivolaropoulos, J. Cosmol. Astropart. Phys. 07 (2008) 012; D. Rubin et al., Astrophys. J. 695, 391 (2009).

[15] S. Nojiri, S. D. Odintsov, and S. Tsujikawa, Phys. Rev. D 71, 063004 (2005)

[16] W. J. Percival et al., Mon. Not. R. Astron. Soc. 381, 1053 (2007).

[17] D. J. Eisenstein and W. Hu, Astrophys. J. 496, 605 (1998).

[18] E. Komatsu et al., Astrophys. J. Suppl. Ser. 180, 330 (2009).

[19] Y. Wang and P. Mukherjee, Phys. Rev. D 76, 103533 (2007); E. L. Wright, Astrophys. J. 664, 633 (2007).

[20] W. Hu and N. Sugiyama, Astrophys. J. 471, 542 (1996).

[21] E. V. Linder and G. Robbers, J. Cosmol. Astropart. Phys. 06 (2008) 004; F. De Bernardis, R. Bean, S. Galli, A. Melchiorri, J. I. Silk, and L. Verde, Phys. Rev. D 79, 043503 (2009). 